\title{
Design and Control of a New Ultrasonic Stepping Motor
}

\author{
K. T. Chau ${ }^{1}$, B. Shi ${ }^{1}$, M. Q. $\mathrm{Hu}^{2}$ and S. W. Chung ${ }^{1}$ \\ ${ }^{1}$ Department of Electrical and Electronics Engineering, The University of Hong Kong, Pokfulam Road, Hong Kong, China \\ ${ }^{2}$ Department of Electrical Engineering, Southeast University, Nanjing 210096, Jiangsu, China
}

\begin{abstract}
In this paper, a systematic approach to design ultrasonic stepping motors (USMs) using spatially shifted standing vibrations is presented, with emphasis on the combination of the order of vibration mode, the number of spatial phase shifts, the number of electrode divisions and the number of sections to excite a mode. Based on the operating principle, the design equations and constraints are mathematically derived, hence proposing the design procedure. The finite element method is also employed to investigate the vibration of the stator. Moreover, by borrowing the idea of electromagnetic stepping motors, the half-step-operation control is newly applied to this USM so that the step size can be further halved. A 80-step USM is prototyped. The proposed design approach and control scheme are well supported by experimentation.
\end{abstract}

Keywords—ultrasonic stepping motor; design; control; drive

\section{INTRODUCTION}

The traveling-wave ultrasonic motor (TWUM) has become attractive for servo applications because of its advantages of high torque at low speed, lightweight, compact size, fast response, no electromagnetic interference (EMI), and quiet operation [1],[2]. Nevertheless, the TWUM needs a closedloop system with precision sensing devices [3]-[6]. In order to get rid of this bulky and costly closed-loop system, the ultrasonic stepping motor (USM) was proposed [7], [8], which involves many projections on the stator and many slits in the rotor as well as the difficulty in controlling the degenerated modes.

Recently, a new type of USMs, using the concept of spatial phase shift of standing vibrations, has been proposed in which the stator does not have any projection [9]. However, the design of the electrode pattern for this USM is ad-hoc. There are different operation cases and different combinations of design parameters for a desired number of steps. Most of them even can not meet the design requirements. So, it is highly desirable to determine which operation is appropriate and which combination is optimum.

The purpose of this paper is to newly propose a systematic approach for the design of USMs using spatially shifted standing vibrations. Different operation cases will be analyzed and the constraint equations will be derived. Based on the presented methods, a new 80-step USMs will be designed, prototyped and tested. The proposed design approach will be supported by simulation and experimentation.

\section{OPERATING PRINCIPLE AND MOTOR CHARACTERISTICS}

As we known, when a small object is put on a vibrating plate, it moves to the nodal position of the vibration and rests at that point [10]. When the nodal position is moved, the object will move too until rest at a new nodal point. The standing wave USM actually utilizes this phenomenon. Fig. 1 shows the realization of the working principle in a ring-type stator of the USM. The electrodes with the same letter identifier are connected together as one phase. It can be seen that when the phase- $\mathrm{A}$ is activated by a high-frequency ac voltage, a standing wave vibration (indicated by a solid curve) is excited so that the small object rests at the nodal point 1 . When the excitation is switched to the phase- $B$, the spatial phase shift of the standing wave vibration (indicated by a dotted line) occurs. Thus, the small object moves from the point 1 to the point 2 , and takes a rest until another phase shift is turned up. The spatial phase shift corresponds to the step angle of the motor. By exciting the phases in sequence, the small object will rotate step by step along the annular stator surface. In the practical USM, the blades of the rotor act as the small objects, which are in contact with the stator surface at points. The direction of the motion can be reversed by changing the shift sequence of the exciting sources.

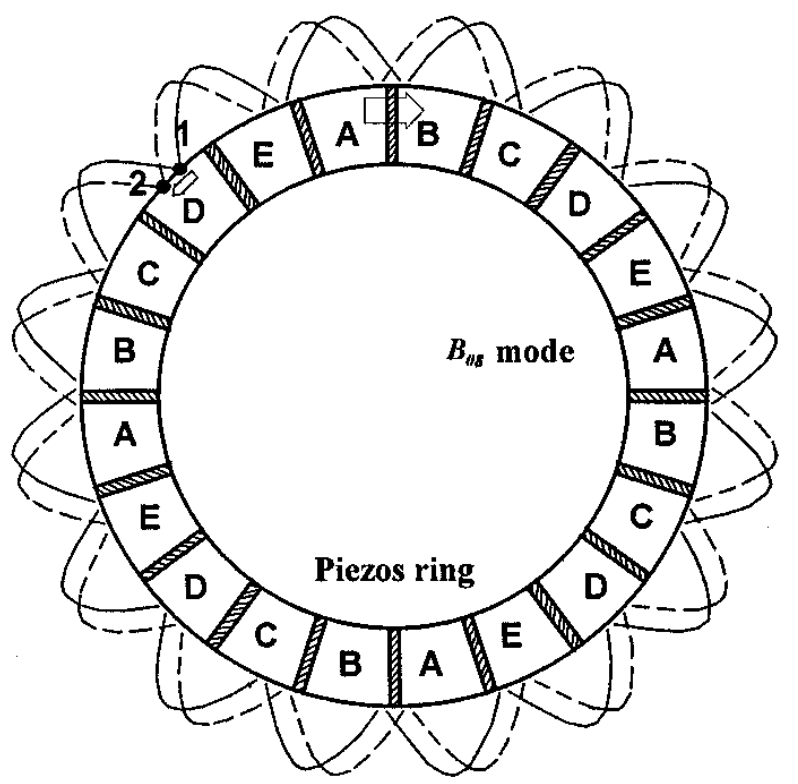

Fig. 1. Step movement realization based on spatial phase shift. 


\section{Design of the Electrode PAtTern}

To design an USM with $N$ steps per revolution, the structures of the stator and the rotor must be determined first. The structure dimension of the stator and the rotor mainly comes from the traditional ring-type ultrasonic motors. So, the design is mainly focused on determining the electrode pattern of the stator and the number of blades on the rotor.

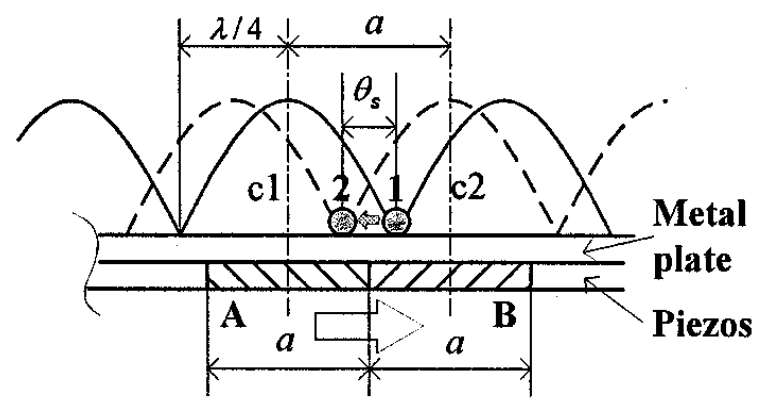

(a)

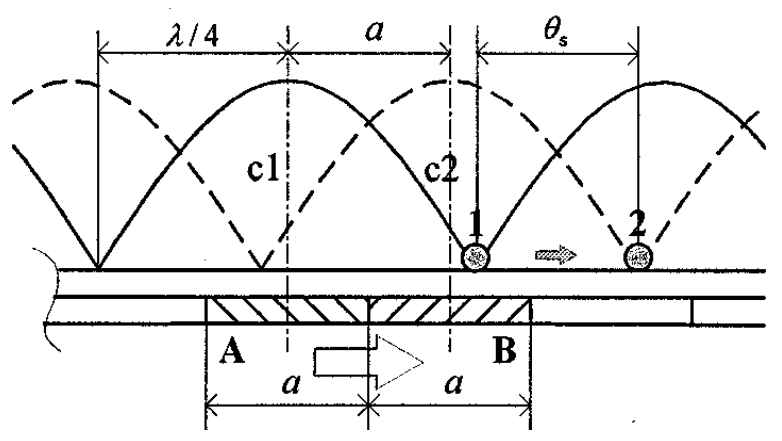

(b)

Fig. 2. Two operation cases. (a) Small step size shift. (b) Large step size shift.

Since the space between the blades of the rotor should be half a wavelength of the stator vibration or its multiple, the number of blades depends on the vibration mode of the stator. So, the key is to design the electrode pattern of the stator, namely the optimum combination of the order of vibration mode $n$, the number of spatial phase shifts (equivalent to the number of driving phases) $p$, the number of electrode divisions $q$ and the number of sections $m$ to excite a mode each time.

Taking into account the principle of moving object by the spatial node phase shift, the ring-type stator with $B_{0 n}$ vibration mode is adopted. Thus, the wavelength $\lambda$ and the length of the divided electrode $a$ can be represented as $\lambda=2 \pi / n$ and $a=2 \pi / q$, whereas the step size can be indicated as $\theta_{s}=2 \pi / N$.

When the exciting source is switched from one phase to the neighbor phase, the spatial phase shift occurs. According to the working principle of the motor, the spatial phase shift should be smaller than the distance from the wave crest to the node just next to it. So, the largest step is smaller than a quarter wavelength of the vibration, $\theta_{s}<\lambda / 4$. Consequently, it yields:

$$
N>4 n
$$

To avoid the unnecessary mechanical damping and generate the expected vibration mode, the length of each electrode should not be greater than a half wavelength. When the length is equal to a half wavelength, the spatial phase shift does not occur. So, it results $a<\lambda / 2$, which can be rewritten as:

$$
q>2 n
$$

Usually, $m$ sections of $q$ electrodes are driven at the same time to excite the required vibration mode $n$. Since these sections are connected together as a common phase, it yields:

$$
q=p \times m
$$

In order to actuate the $B_{0 n}$ vibration mode effectively, $m$ electrodes should be arranged symmetrically around the circumference. To match with the stator vibration mode, $m$ must be an integer divisor of the mode number $n$.

For a $N$ step ultrasonic motor, the step size is certainly determined by $\theta_{s}=2 \pi / N$. The design is to find the appropriate and optimum combination of the vibration mode, electrode divisions and the number of driving phases to make the step size equal to $\theta_{s}$. Before implementing the desired step size $\theta_{s}$, the relationship between the step size and the length of the electrode should be examined. In accordance with different relative lengths of the electrode $a$, there are two different operation cases.

\section{A. Small step size}

Fig. 2(a) shows the case when the length of the electrode is greater than a quarter wavelength, $a>\lambda / 4$ (equivalent to $q<4 n)$. When the driving source is switched from the phaseA to the phase- $B$, the spatial phase of the standing wave vibration is shifted from the position indicated by the solid curve to the position by the dashed curve. The object on the surface moves from node 1 to node 2 at the same time. The direction of the movement is opposite to the shift of the driving phase. As shown in Fig. 2(a), the step size from node 1 to node 2 can be calculated as the overlapping width of two quarters wave. So, the step size $\theta_{s}$ can be expressed as:

$$
\theta_{s}=\lambda / 4+\lambda / 4-a=\lambda / 2-a=(\pi / n-2 \pi / q)
$$

Since $\theta_{s}=2 \pi / N$, the electrode divisions $q$ can be calculated as:

$$
q=\frac{2 n N}{N-2 n}
$$

Since the corresponding derivative of $q$ with respect to $n$ is always positive, the function $q$ is monotonically increasing with respect to $n$. In other words, only one value of $q$ practically satisfies the design objectives for a particular vibration mode $n$. Equation (5) can be treated as the design criterion to determine the electrode divisions corresponding to the vibration mode.

As shown in Fig. 2(a), the shift distance of each phase switching is equal to the length of one electrode $a$. After $p$ times switching, the shift distance can be calculated as $p \cdot a=p \cdot 2 \pi / q=2 \pi / m$. The phase-A is excited again and 
the standing-wave vibration is again indicated by the solid curve as shown in Fig 2(a). So, the object moves from the previous node line to the node line of the same standing-wave vibration in the near-by region, namely the object moves a distance of one or several half-wavelengths. Thus, one of the following relationships is valid:

$$
\begin{cases}N=2 n \times p & \text { for } \lambda / 2 \\ N=n \times p & \text { for } \lambda \\ N=n \times p \times 2 / 3 & \text { for } 3 \lambda / 2 \\ N=n \times p / 2 & \text { for } 2 \lambda\end{cases}
$$

Since a particular movement distance corresponds to a particular number of spatial phase shifts, $p$ can be determined by:

$$
\begin{cases}p=N / n / 2 & \text { for } \lambda / 2 \\ p=N / n & \text { for } \lambda \\ p=N / n \times 3 / 2 & \text { for } 3 \lambda / 2 \\ p=N / n \times 2 & \text { for } 2 \lambda\end{cases}
$$

Therefore, for a pre-chosen vibration mode $n$, there is only one electrode division $q$ that satisfies the $N$ steps design requirement, according to (5). However, there are different possible driving phase shifts $p$ as shown in (7). In practice, the number of the phase shifts $p$ should be made as small as possible to simplify the driving and control circuits. So, only the first equation in (7) is considered:

$$
\begin{aligned}
& p=N / 2 n \\
& m=q / p
\end{aligned}
$$

Based on the above equation from (5) to (9), there is only one set of $q, p, m$ corresponding to the vibration mode $n$ that satisfies the design requirement.

For example, due to the lack of systematic design approach and criterion, Nakamura [9] proposed 9 possible combinations of $n, q, p$ (shown in Table 1) for a 60-step USM. However, based on the proposed design criterion, only two combinations are appropriate. The actual step sizes are also given in Table 1. The reason to this incorrect design is a result of ignoring the other operation case as illustrated below.

\section{B. Large step size}

Fig. 2(b) shows another operation case that the length of the electrode is less than or equal to a quarter wavelength ( $a \leq \lambda / 4$, namely $q \geq 4 n$ ). When the driving source is switching from the phase-A to the phase-B, the spatial phase of the standing wave vibration and the object move similar to the previous case. However, the movement direction of the object is same as the shift of the driving phase. As shown in Fig. 2(b), the step size from node 1 to node 2 can be calculated by using the centerline of the A-electrode as the reference:

$$
\theta_{s}=a+\lambda / 4-\lambda / 4=a
$$

Since $\theta_{s}=2 \pi / N$ and $a=2 \pi / q$, the division $q$ is given by:

$$
q=N
$$

As shown in Fig. 2(b), the shift distance of each phase switching is equal to the length of one electrode $a$ and the step size $\theta_{s}$. So, after $p$ times switching, the shift distance of driving phase is $2 \pi / m$. The phase-A is excited again and the standing-wave vibration is again indicated by the solid curve. The movement distance of the object is also $2 \pi / \mathrm{m}$, which is of one or several wavelengths. So, the following relationships arises:

$$
\begin{cases}N=n \times p & \text { for } \lambda \\ N=n \times p / 2 & \text { for } 2 \lambda\end{cases}
$$
by:

So the number of spatial phase shifts $p$ can be calculated

$$
\begin{cases}p=N / n & \text { for } \lambda \\ p=N / n \times 2 & \text { for } 2 \lambda\end{cases}
$$

Based on the same reason described in the previous case, only the first equation in (13) is considered to calculate $p$ :

$$
p=N / n
$$

Since $q=p \times m$ and $q=N$, it yields:

$$
m=n
$$

Looking through the given combinations in Table I again, all the incorrect designs correspond to the this operation case when the relationship $q \geq 4 n$ holds true. Namely, their step numbers $N$ are equal to the electrode divisions $q$.

TABLE I. DESIGN Of 60-STEP USM USING Proposed CRITERION

\begin{tabular}{ccccccc}
\hline$n$ & $q$ & $p$ & $m$ & $q<4 n$ & $q=\frac{2 n N}{N-2 n}$ & $\begin{array}{c}\text { Actual } \\
\text { steps }\end{array}$ \\
\hline 1 & 30 & 30 & 1 & NO & NO & 30 \\
2 & 15 & 15 & 1 & NO & NO & 15 \\
2 & 30 & 15 & 2 & NO & NO & 30 \\
3 & 10 & 10 & 1 & YES & NO & 15 \\
3 & 30 & 10 & 3 & NO & NO & 30 \\
5 & 30 & 6 & 5 & NO & NO & 30 \\
6 & 15 & 5 & 3 & YES & YES & 60 \\
6 & 30 & 5 & 6 & NO & NO & 30 \\
10 & 30 & 3 & 10 & YES & YES & 60 \\
\hline
\end{tabular}

TABLE II. COMPARISON OF TWO DIFFERENT OPERATION CASES

\begin{tabular}{cc|cc|cc}
\hline & & \multicolumn{2}{|c|}{ Small step case } & \multicolumn{2}{c}{ Large step case } \\
$N$ & $n$ & $q$ & $p$ & $q$ & $p$ \\
\hline 20 & 2 & 5 & 5 & 20 & 10 \\
30 & 5 & 15 & 3 & 30 & 6 \\
48 & 8 & 24 & 3 & 48 & 6 \\
60 & 6 & 15 & 5 & 60 & 10 \\
80 & 8 & 20 & 5 & 80 & 10 \\
\hline
\end{tabular}




\section{Comparison of two cases}

Theoretically, the design criterion to find the appropriate combination of $n, q, p$ can be based on the above two operation cases. However, the design based on the second case has less practicability. Since the number of electrode divisions $q$ is equal to the number of steps $N, q$ must be large enough to get a smaller step size, which makes the electrode division of piezoelectric ceramic very difficult and costly. Furthermore, the interconnection between small electrode sections is also complicated. Comparing the two designs with the same step size and vibration mode as summarized in Table II, it is obvious that the design based on the second case has double phase shifts $p$. The drive circuit will be more complicated and costly. So, the second design criterion of the large step size case will only be used for some special cases such as the requirement of odd step numbers which can not be implemented by the first design criterion of the small step size case.

When the first design criterion is utilized, there are always many combinations that are considered appropriate. Taking into account of the fact that the higher order vibration mode usually causes lower amplitude and can not be used to drive the rotor effectively, the low-order vibration mode is usually preferred. This selection also results in the less number of electrode divisions, due to the monotonic increasing function relationship between $n$ and $q$.

\section{Design procedure}

Step 1: The desired number of steps $N$ is given. The initial value of the vibration mode is set to 1 .

Step 2: If the value of $n$ is greater than a quarter of $N$, there is no combination based on the small step size operation case for the given step number $N$, and the design can be proceeded based on the larger step size operation case (go to Step 5). Otherwise, the design continues to step 3.

Step 3: The electrode divisions $q$, the number of driving phases $p$ and the electrodes to excite the vibration mode included in a phase $m$ are calculated on the basis of the design criterion given by (5), (8), (9).

Step 4: When the values of $q, p, m$ are all integers and $m$ is the factor of $n$, the desired design combination is obtained and the design is completed. Otherwise, after increasing the value of $n$ by 1, the process jumps to Step 2 .

Step 5: If the requirement of step number can not be satisfied based on the small step size operation case, one can take the design based on the large step size operation case. Thus, (11), (14), (15) are used as the design criterion. It must be noted that the division of the electrodes and the number of driving phases are usually large in this case. Due to the difficulties in manufacturing the piezoelectric plates and interconnecting different sections of electrodes, the corresponding number of steps can not be too large, for example, less than 40 . In this circumstance, the number of driving phases $p$ can be selected as a constraint, and there is a trade-off between the selection of $p$ and $n$.
Table III shows some design examples for an USM with the step requirements from 20 to 100 based on the small step size operation case.

\section{IMPLEMENTATION OF The PROTOTYPE}

Based on the proposed design approach, a new 80-step USM has been designed and built. The corresponding structure is shown in Fig. 3. Its geometric parameters are tabulated in Table IV. Phosphor bronze is selected as the material of the elastic ring of the stator. Its elastic modulus is also insensitive to the varying temperature [11]. The piezoelectric ceramic ring has 20 electrode divisions etched on it. As shown in Fig. 1, the electrodes with the same letter are connected together as a driving phase. The rotor is made of aluminum because of lightness and easy machining. There are 16 blades machined at the contact surface with the stator. The elastic caoutchouc between the flange of the shaft and the rotor acts as a fine-tuned spring. The prototype of the 80 -step USM is also shown in Fig. 4.

TABLE III. DESIGN OF USM With 20 To 100 StePS

\begin{tabular}{|c|c|c|c|c|}
\hline$N$ & $n$ & $q$ & $p$ & $m$ \\
\hline 20 & 2 & 5 & 5 & 1 \\
\hline 24 & 4 & 12 & 3 & 4 \\
\hline 30 & 5 & 15 & 3 & 5 \\
\hline 36 & 6 & 18 & 3 & 6 \\
\hline 40 & 4 & 10 & 5 & 2 \\
\hline 42 & 3 & 7 & 7 & 1 \\
\hline 42 & 7 & 21 & 3 & 7 \\
\hline 48 & 8 & 24 & 3 & 8 \\
\hline 54 & 9 & 27 & 3 & 9 \\
\hline 60 & 6 & 15 & 5 & 3 \\
\hline 60 & 10 & 30 & 3 & 10 \\
\hline 66 & 11 & 33 & 3 & 11 \\
\hline 72 & 4 & 9 & 9 & 1 \\
\hline 72 & 12 & 36 & 3 & 12 \\
\hline 78 & 13 & 39 & 3 & 13 \\
\hline 80 & 8 & 20 & 5 & 4 \\
\hline 84 & 6 & 14 & 7 & 2 \\
\hline 84 & 14 & 42 & 3 & 14 \\
\hline 90 & 15 & 45 & 3 & 15 \\
\hline 96 & 16 & 48 & 3 & 16 \\
\hline 100 & 10 & 25 & 5 & 5 \\
\hline
\end{tabular}

TABLE IV.

PHYSICAL PARAMETER OF PROTOTYPE USM

\begin{tabular}{ll}
\hline Inner radius of stator & $22.5 \mathrm{~mm}$ \\
Outer radius of stator & $30.0 \mathrm{~mm}$ \\
Height of stator & $3.0 \mathrm{~mm}$ \\
Height of piezos & $0.5 \mathrm{~mm}$ \\
\hline
\end{tabular}




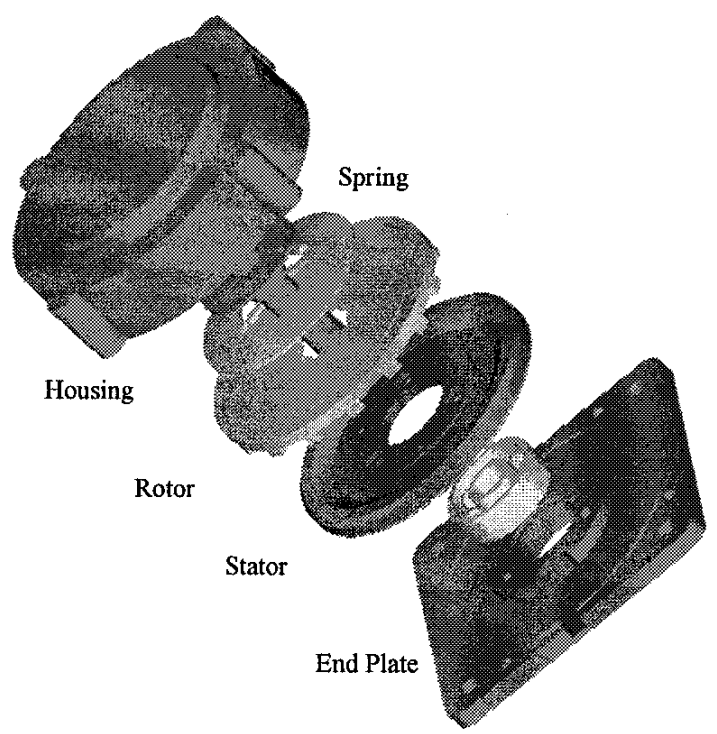

Fig. 3. Structure of USM.

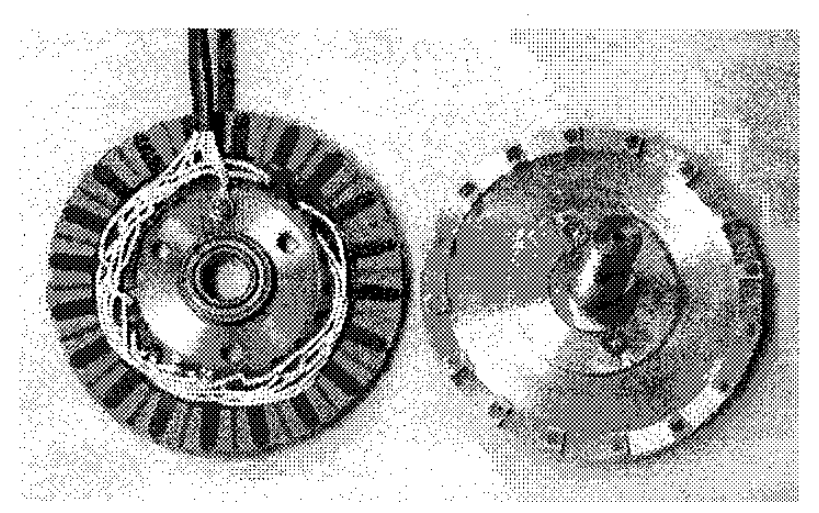

Fig. 4. Prototype of 80 -step USM (left: stator; right: rotor).

The vibration mode analysis is conducted to find the appropriate driving frequency to excite the desired vibration. A finite element method (FEM) based on the commercially available software ANSYS is utilized. The coupling element is used to model the stator including the piezoelectric plates [12]. The analysis result is depicted in Fig. 5. It can be seen that the $B_{08}$ vibration mode is implemented. The calculated resonant frequency is $44.9 \mathrm{kHz}$.

In order to drive the motor effectively, the practical resonant frequency must be measured. According to the equivalent circuit of the ultrasonic motors [13], the resonant frequency can be obtained by the impedance measurement of the stator. Fig. 6 shows the impedance of the stator. The minimum amplitude of the impedance corresponds to the resonant frequency point, which is found to be of $40.36 \mathrm{kHz}$. The difference between the calculated value and the measured value is usually due to the discrepancy between the theoretical and practical data of the piezoelectric material.
The driving circuitry is shown in Fig. 7. The VCO module unit acts as the driving frequency generator. The highfrequency AC signal is modulated by the driver phase shift control, which determines the sequence and the direction of the phase switching. The 5-phase power driver consists of the MOSFET and boost transformer so as to provide a high voltage to drive the piezoelectric plates. The pulse generator controls the speed of the motor. When there is one pulse input, the phase switches one time and one step-size movement of the rotor arises. To be flexible in control and measurement, the communication unit with the PC is also integrated into the circuit.

To verify the stepping shift of the spatial phase, the vibration mode of the stator is measured by using an electronic speckle pattern interferometer [14]. Fig. 8(a) shows the spatial distribution of the vibration when only the phase-A is excited. The gray dark lines along the circumference denote the nodal diameters. The reference position of one nodal diameter is indicated by a dashed line marked with 'Ref'. When the driving source is switched from the phase-A to the phase-B, the spatial phase shift occurs. Fig. 8(b) shows the spatial distribution when only the phase-B is driven. The corresponding nodal diameter is thus indicated by a solid line. Comparing the two patterns, the spatial phase shift is $4.5^{\circ}$ when the exciting source is switched from the phase-A to the phase-B. So, the blades of the rotor also moves $4.5^{\circ}$ degree, totally 80 steps per revolution.

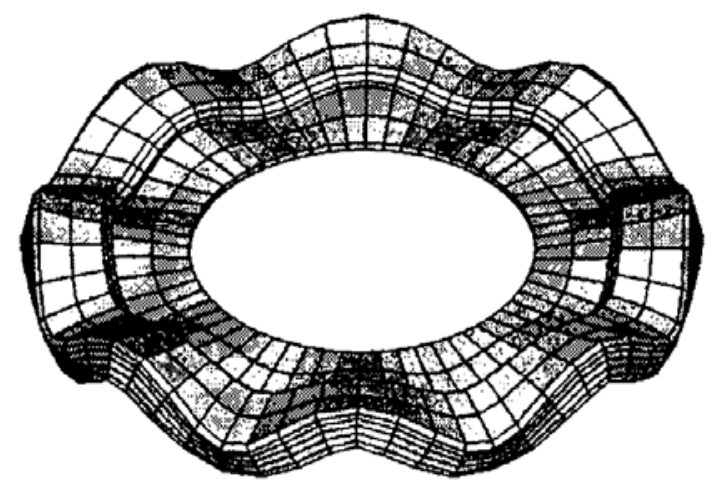

Fig. 5. Vibration mode analysis using FEM

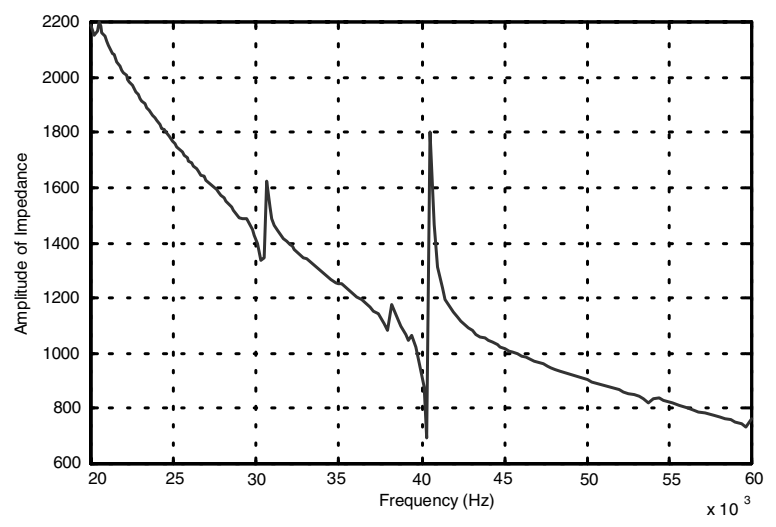

Fig. 6. Measured impedance of stator. 


\section{CHARACTERISTICS}

A measurement system is constructed to investigate the characteristics of the prototype. The driving circuit is controlled by the host PC. The on/off, CW/CCW, the step speed commands are transmitted to the driver through the LPT port. The motor is connected with a encoder with $2500 \mathrm{ppr}$ to get the angular position of the rotor. A DSP microcontroller TMS320F240 is employed to handle the position and speed information from the encoder pulses.

The driving frequency is set to the resonant frequency of the stator, namely $40.36 \mathrm{kHz}$. The driving voltage on the sections of the piezoelectric ceramics is set to 200Vpp. During the experiment, the position information is sent to the host PC by the DSP. The sampling frequency of the position is set to $400 \mathrm{~Hz}$.

Fig. 9 shows the transient response of the rotor rotation when the driving source is switching from one phase to the other. The step movement is implemented within $0.2 \mathrm{~s}$. The speed arises very quickly to a high level and slow down gradually.

The step rotation characteristics are measured. The movement command is programmed in the host PC. After 10 steps rotation in the clockwise direction, the rotation is reversed to get another 10 steps. The rotor returns to its original position. As shown in Fig. 10, the step size is $4.5^{\circ}$. Hence, the proposed design approach of the 80-step USM is successfully verified by experiment. During the operation, the maximum deviation is less than $1^{\circ}$, and there is no cumulative error.

As we known, for the traditional electromagnetic stepping motor, the half-step operation can be realized by combining the single-phase and two-phase excitations. Extending this idea to USMs, the half-step operation excitation scheme is proposed for this USM.

Table $\mathrm{V}$ shows the corresponding excitation sequences. The single-phase excitation corresponds to the normal singlephase-on operation. The combined phase excitation corresponds to the half-step operation. Fig. 11 shows the step characteristics under half-step operation. Besides the half-step movement of the rotor, an interesting phenomenon can be observed. Namely, the speed of the rotor is changed rapidly. It This is because that the excitation is switching between onephase to two-phase. Fig. 12 shows the transient movement of the rotor when the excitation moves from one phase to two phases under half-step operation. Comparing the results in Fig. 9 , the rotor can reach the static position within $0.06 \mathrm{~s}$, which is much faster than that of normal operation.

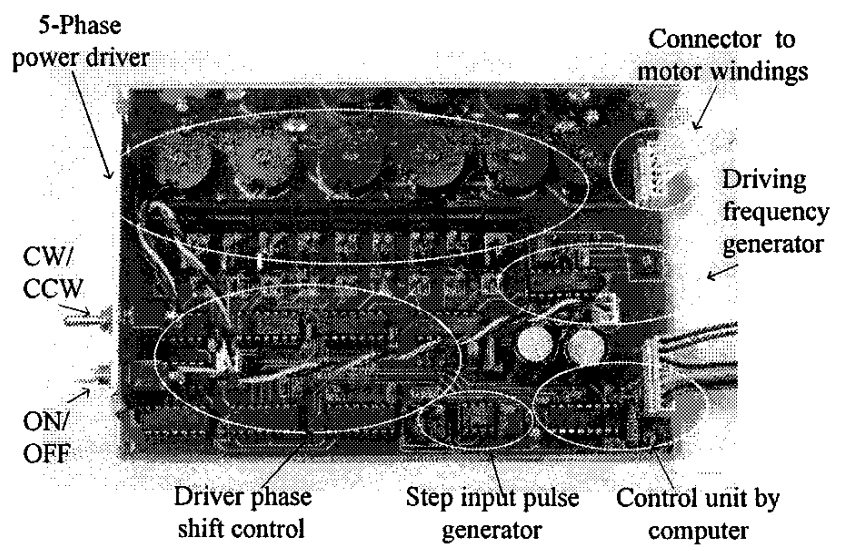

Fig. 7. Driving circuit of the stepping ultrasonic motor.

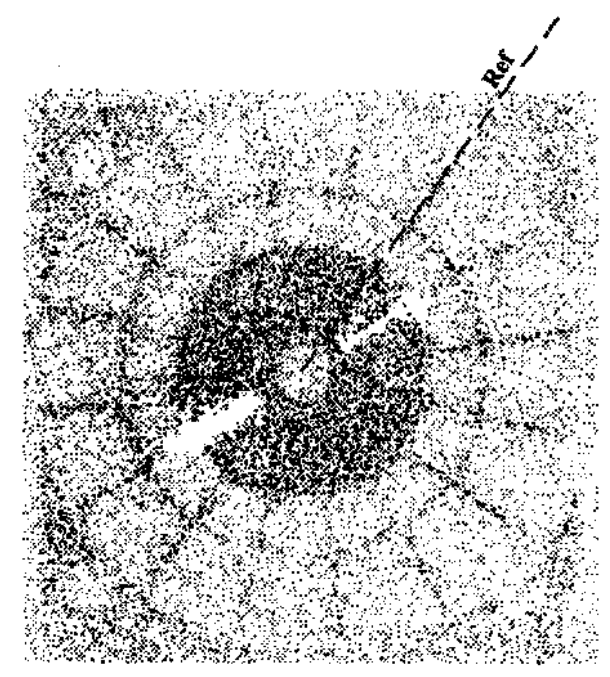

(a)

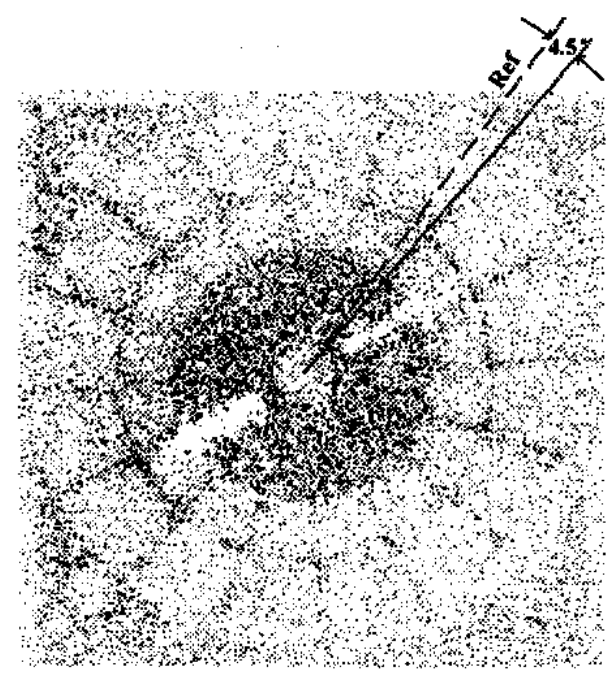

(b)

Fig. 7. Measured vibration mode. (a) Phase-A excited. (b) Phase-B excited. 


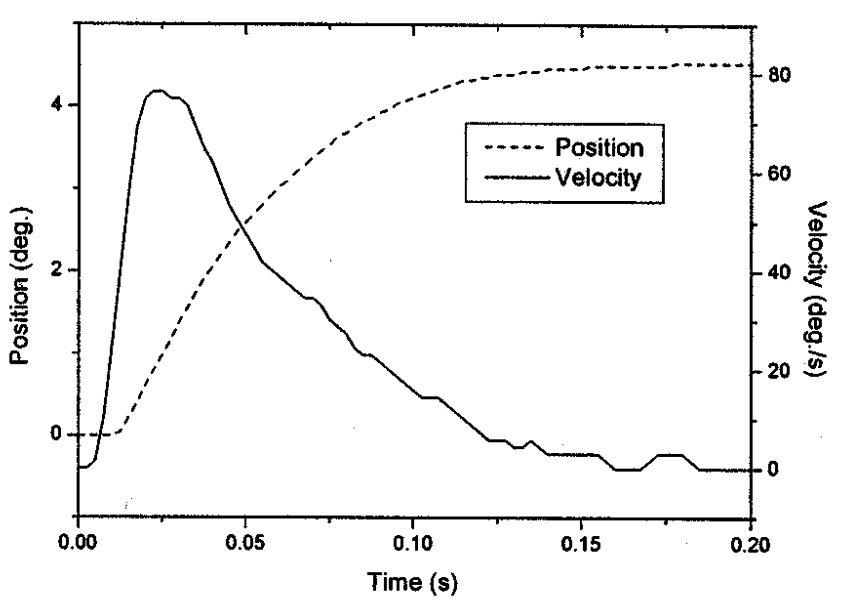

Fig. 9. Transient response of the rotor rotation.
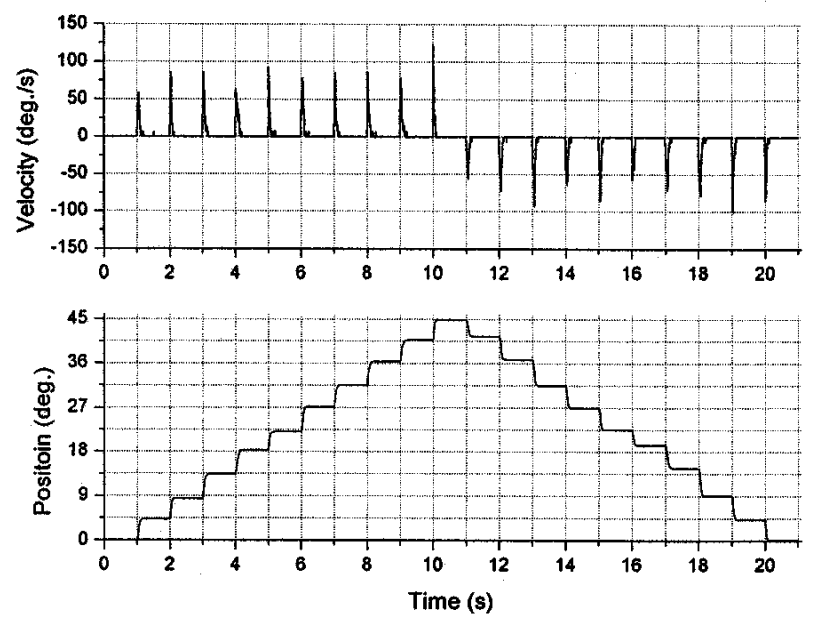

Fig. 10. Rotor step movement characteristics under full-step operation.

TABLE V. EXCITATION SEQUENCES

Single-phase-on operation

\begin{tabular}{|l|l|l|l|l|l|l|l|l|l|l|}
\hline Clock state & $\mathrm{R}$ & 1 & 2 & 3 & 4 & 5 & 6 & 7 & 8 & 9 \\
\hline Phase A & & & & & & & & & & \\
\hline Phase B & & & & & & & & & & \\
\hline Phase C & & & & & & & & & & \\
\hline Phase D & & & & & & & & & & \\
\hline Phase E & & & & & & & & & & \\
\hline
\end{tabular}

Half-step operation

\begin{tabular}{|l|l|l|l|l|l|l|l|l|l|l|}
\hline Clock state & $\mathrm{R}$ & 1 & 2 & 3 & 4 & 5 & 6 & 7 & 8 & 9 \\
\hline Phase A & & & & & & & & & & \\
\hline Phase B & & & & & & & & & & \\
\hline Phase C & & & & & & & & & & \\
\hline Phase D & & & & & & & & & & \\
\hline Phase E & & & & & & & & & & \\
\hline
\end{tabular}
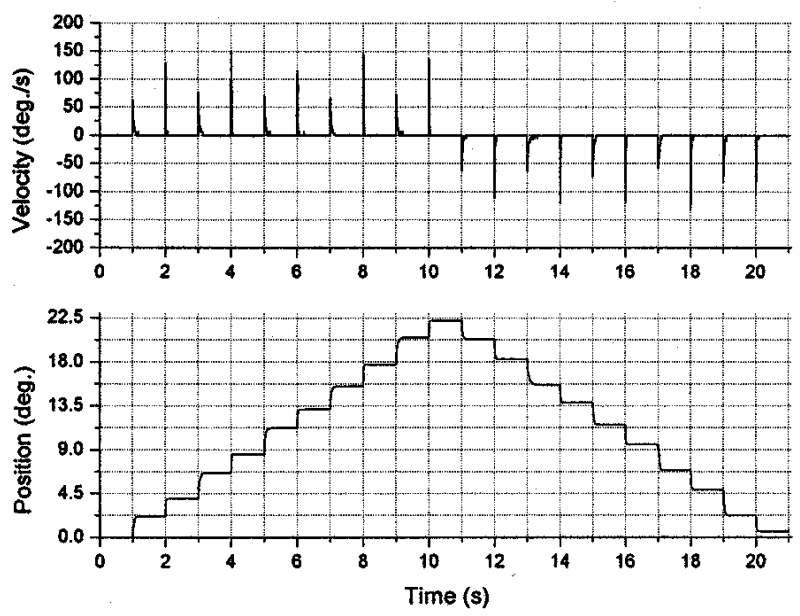

Fig. 11. Rotor step movement characteristics under half-step operation.

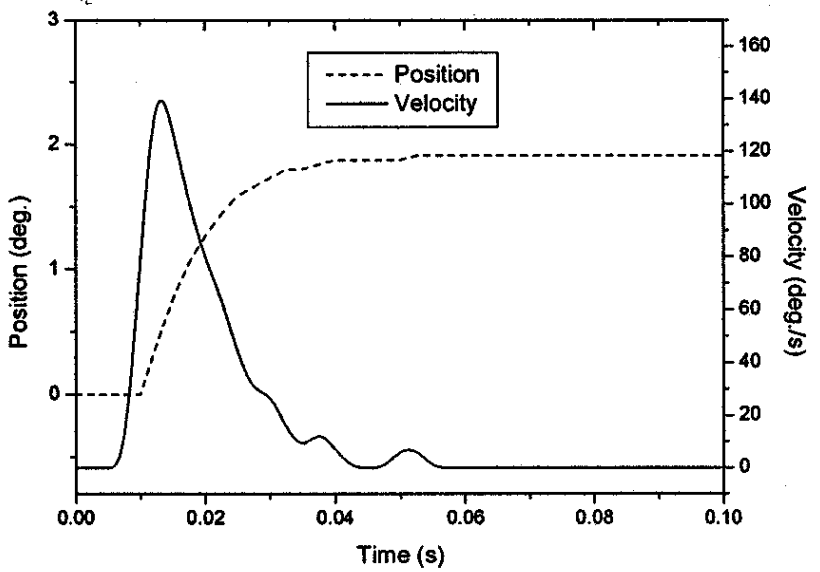

Fig. 12. Transient response of the rotor rotation under half-step operation.

\section{CONCLUSION}

A systematic design approach for USMs using spatially shifted standing vibrations has been presented. The design equations governing the combination of the order of vibration mode, the number of spatial phase shifts, the number of electrode divisions and the number of sections to excite a mode have been derived, hence proposing the design procedure. The finite element method has also been employed to investigate the vibration of the stator. A 80-step USM has been designed, prototyped and tested. The proposed design approach is well supported by experimentation. Following the control idea adopted by electromagnetic stepping motors, the half-step-operation control scheme is also newly applied to this USM and verified by experiments.

\section{ACKNOWLEDGMENT}

This work was supported and funded by the Hong Kong Research Grants Council (Project No. HKU 7039/00E). 


\section{REFERENCES}

[1] S. Ueha, and Y. Tomikawa, Ultrasonic motors: Theory and Applications. New York: Oxford Science Publications, 1993.

[2] K. Uchino, "Piezoelectric ultrasonic motors: overview," Smart Materials and Structures, Vol. 7, No. 3, 1998, pp. 273-285.

[3] T. Senjyu, H. Miyazato, and K. Uzeato, "Quick and precise position control of an ultrasonic motor with dual mode control," International Journal of Electronics, Vol. 80, No. 2, 1996, pp. 191-200.

[4] F.J. Lin, "Fuzzy adaptive model-following position control for ultrasonic motor," IEEE Transactions on Power Electronics, Vol. 12, No. 2, 1997, pp. 261-268.

[5] K.T. Chau, and S.W. Chung, "Servo position control of ultrasonic motors using fuzzy neural network," Electric Machines and Power Systems, Vol. 29, No. 3, 2001, pp. 229-246.

[6] K.T. Chau, and S.W. Chung, "Servo Speed Control of Traveling-Wave Ultrasonic Motors using Pulse Width Modulation," Electric Power Components and Systems, Vol. 29, No. 8, 2001, pp. 707-722.

[7] C. Xiaoduo, "Rotor displacement of the ultrasonic motor having an angular displacement self-correction function," Japanese Journal of Applied Physics, Vol. 32, Part 1, No. 9B, 1993, pp. 4198-4201.
[8] C. Kusakabe, "Effect of pressing force applied to a rotor on disk-type ultrasonic motor driven by self-oscillation," Japanese Journal of Applied Physics, Vol. 37, Part 1, No. 5B, 1998, pp. 2966-2969.

[9] K. Nakamura, J. Margairaz, T. Ishii, and S. Ueha, "Ultrasonic stepping motor using spatially shifted standing vibrations," IEEE Transactions on Ultrasonics, Ferroelectrics and Frequency Control, Vol. 44, No. 4, 1997, pp. 823-827

[10] A.W. Leissa, Vibration of plates. Washington, D.C.: Scientific and Technical Information Division, National Aeronautics and Space Administration, 1969.

[11] T. Sashida, and T. Kenjo, An introduction to ultrasonic motors. New York: Oxford Science Publications, 1993.

[12] ANSYS Theory Reference, Release 5.5, 9th Editions. SAS IP, Inc.

[13] N.E. Ghouti, Hybrid Modeling of a Traveling Wave Piezoelectric Motor. Doctoral thesis, Aalborg University, Denmark, 2000.

[14] J. Shu-hai, Y. Kai-duan, and T. Yu-shan, "The development of espi for vibration measurment," Chinese Journal of Applied Optics, Vol. 20, No. 4, 1999, pp. 41-45. 